\title{
A INTERDISCIPLINARIDADE DA ENGENHARIA AMBIENTAL SOB A ÓTICA DO ESTUDANTE DE GRADUAÇÃO: CORRELACIONANDO DISCIPLINAS DO CURSO COM CONDICIONANTES AMBIENTAIS
}

\author{
Marina dos Santos Oliveira - marina.santos1@aluno.ufop.edu.br \\ Universidade Federal de Ouro Preto, Escola de Minas. \\ Campus Universitário, Morro do Cruzeiro, $s / n$. \\ CEP 35400-000 - Ouro Preto - MG
}

Guilherme José Cunha Gomes - guilhermejcg@ufop.edu.br

Universidade Federal de Ouro Preto, Escola de Minas.

Campus Universitário, Morro do Cruzeiro, $s / n$.

CEP 35400-000 - Ouro Preto - MG

Resumo: Os cursos de engenharia são essencialmente interdisciplinares, refletindo as necessidades e desafios da condução de projetos complexos do mundo real. Dentre os cursos de graduação em engenharia, a Ambiental é uma das dependentes da interdisciplinaridade. Neste trabalho, os alunos da disciplina AMB 176 - Avaliação de Impacto e Licenciamento Ambiental, do curso de Engenharia Ambiental da Universidade Federal de Ouro Preto (UFOP), fazem um levantamento de como as disciplinas ofertadas no curso se relacionam com as condicionantes ambientais impostas nos processos de licenciamento. Condicionantes ambientais de oito diferentes empreendimentos sujeitos a Estudo de Impacto Ambiental (EIA) conforme a Resolução CONAMA 01/86 são avaliadas. Os alunos foram direcionados a fazer um paralelo entre as disciplinas obrigatórias e eletivas do curso necessárias ao atendimento das exigências dos órgãos licenciadores. Os resultados demonstraram que disciplinas da área de ciências biológicas e da área de gestão ambiental, foram apontadas como mais relevantes pelos discentes. Dentre as sete necessidades apontadas pelos alunos como prérequisitos para o atendimento de condicionantes ambientais, quatro delas ainda não são ofertadas pelo curso. As cinco disciplinas do curso relevantes (comuns) para todos os empreendimentos são oferecidas por diversos departamentos dentro da Universidade, refletindo o caráter interdisciplinar do curso de Engenharia Ambiental. Das cinco disciplinas do curso relacionadas a todas as licenças ambientais, a disciplina FAR 402 - Qualidade das Águas é não-obrigatória (eletiva) atualmente no curso de Engenharia Ambiental.

Palavras-chave: Interdisciplinaridade. Engenharia Ambiental. Licenciamento Ambiental. Condicionantes Ambientais.

\section{INTRODUÇÃO}

O modelo tradicional de ensino dos cursos de engenharia, baseados em monodisciplinaridade, principalmente nas etapas básicas, que envolvem disciplinas das ciências exatas e biológicas, assume que os estudantes de graduação irão trabalhar como especialistas (Harrison, 2007). Como consequência, muitos estudantes encontram dificuldade em conectar as diversas disciplinas dos cursos, gerando desafios para o ensino da engenharia na atualidade. Além disso, as empresas atualmente buscam profissionais cada vez mais multi-funcionais, capazes de liderar equipes, conduzir projetos complexos e, mais recentemente, capacitados a trabalhar com a ciência de dados e inteligência artificial em situações reais do cotidiano (Araujo et al., 2020). Esses desafios do mercado e a mudança no perfil dos profissionais 
levaram muitos pesquisadores a tentar desenvolver instrumentos pedagógicos multidisciplinares em diversos cursos, incluindo, dentre outras, as engenharias química (Foley, 2016), elétrica (Guider et al., 2012), civil (Zhang et al., 2020) e ambiental (Semerjian et al., 2004).

No âmbito da engenharia ambiental, observa-se que a interdisciplinaridade é uma peça chave para o desenvolvimento do pensamento crítico por parte do aluno quanto à tomada de decisão (Fortuin, et al., 2011). Devido à sua interconexão com situações que ultrapassam as fronteiras de uma gama enorme de temáticas, diversos profissionais propuseram estudos interdisciplinares no âmbito da engenharia ambiental, envolvendo as disciplinas introdução à engenharia ambiental (Lu, 2015), físico-química (Butkus, 2004), sistemas de informação geográfica (Rickles et al., 2017), gestão ambiental (Bunrley et al., 2019), dentre outras. No Brasil, os cursos de engenharia ambiental ainda contam disciplinas relacionadas com o licenciamento e avaliação de impactos ambientais, que, além de representarem uma porção grande do mercado de trabalho, requerem, por natureza, a interação com muitas outras disciplinas do curso.

O licenciamento ambiental é um importante instrumento de regulação de empreendimentos e uma das principais áreas de atuação para os engenheiros ambientais. Esta, sem dúvidas, é uma das disciplinas mais importantes no processo de formação profissional, demonstrando ao discente como a execução de projetos solicita uma visão ampla a cerca dos temas abordados durante a graduação. Contudo, alunos de graduação carecem de entendimentos sobre como executar projetos de consultoria (técnica e administrativamente) ou como lidar com as tratativas de Órgãos Ambientais nas suas mais diversas esferas. Para tentar entender esta lacuna, RODRIGUES (2010) utilizou pareceres elaborados para emissão de Licença Prévia de empreendimentos de grande porte do setor sucroalcooleiro no Estado de Minas Gerais. A tentativa foi para elucidar a contribuição do modelo interdisciplinar para a formulação de novas estratégias e racionalidades para a avaliação de impactos ambientais. O estudo concluiu que, para que haja um trade-off entre os interesses do empreendedores e os da sociedade, como usuária do meio ambiente, é preciso que a análise de processos seja feita por profissionais capacitados, com espírito multi-disciplinar. Assim, apresentar aos alunos o teor dessa interdisciplinaridade por meio da análise de licenças e de condicionantes ambientais pode ser uma excelente ferramenta didática para os cursos de engenharia ambiental.

Os alunos da disciplina AMB 176 - Avaliação de Impacto e Licenciamento Ambiental, do curso de Engenharia Ambiental da Universidade Federal de Ouro Preto (UFOP), ainda não conhecem os desafios interdisciplinares que envolvem o atendimento de condicionantes de licenças ambientais. Além disso, não se sabe quais as disciplinas do curso são preponderantes para a aquisição de competências exigidas pelo mercado de trabalho. Portanto, este artigo tem por objetivo demonstrar, através do estudo de caso levantado em sala de aula, a ótica dos discentes a respeito da interdisciplinaridade do curso de Engenharia Ambiental da Universidade Federal de Ouro Preto e como as disciplinas ofertadas se relacionam com as condicionantes ambientais impostas nos processos de licenciamento. Neste trabalho, condicionantes ambientais de oito diferentes empreendimentos sujeitos a Estudo de Impacto Ambiental (EIA) conforme a Resolução CONAMA 01/86 são avaliadas. Os alunos foram direcionados a fazer um paralelo entre as disciplinas obrigatórias e eletivas do curso necessárias ao atendimento das exigências dos órgãos licenciadores. As principais questões a serem abordadas no artigo são:

i. Quais áreas do conhecimento (disciplinas do curso) são mais relevantes para o atendimento dessas condicionantes ambientais?

ii. Quais seriam as eventuais "necessidades" para o atendimento dessas condicionantes ambientais? 
iii. Quais áreas do conhecimento (disciplinas do curso) seriam relevantes (comuns) para todos os empreendimentos?

iv. Existem disciplinas relevantes (comuns) a todos os empreendimentos que seriam eletivas atualmente?

\section{METODOLOGIA}

A fim de determinar sob a perspectiva dos discentes as disciplinas mais relevantes ao entendimento e execução das condicionantes propostas pelos órgãos ambientais responsáveis no processo de licenciamento, foram avaliados oito empreendimentos classificados como de grande porte em várias regiões do país como mostra a relação feita na Tabela 1. As licenças ambientais dos empreendimentos listados foram obtidas na internet, pelo professor do curso, e disponibilizadas aos grupos de alunos.

Tabela 1 - Listagem dos empreendimentos analisados neste trabalho, incluindo .

\begin{tabular}{|c|c|c|c|}
\hline Empreendimento & $\begin{array}{c}\text { Licença } \\
\text { Ambiental }\end{array}$ & Local & $\begin{array}{c}\text { Condicionantes } \\
\text { analisadas }\end{array}$ \\
\hline Lavra e Beneficiamento de Cobre & LP 232/2006 & Carajás, PA & 27 \\
\hline Dragagem Portuária & LP 290/2008 & Santos, SP & 17 \\
\hline UHE Belo Monte & LI 795/2011 & Altamira, PA & 16 \\
\hline Implantação Porto Central & LI 1203/2018 & P. Kennedy, ES & 30 \\
\hline Metro de São Paulo & LI 2352/2009 & São Paulo, SP & 14 \\
\hline Ramal Ferroviário & LP 432/2012 & Parauapebas, PA & 31 \\
\hline Gasoduto marítimo e terrestre & LI 1237/2018 & Santos, SP e RJ & 40 \\
\hline Estrada de rodagem & LI 007/2018 & Brasília, DF & 38 \\
\hline \multicolumn{3}{|c|}{ Total de condicionantes avaliadas } & 213 \\
\hline
\end{tabular}

Fonte: Os autores.

Para isso, elaboraram-se grupos de quatro (04) alunos e a cada grupo foi destinado aleatoriamente uma licença ambiental - sendo as mesmas prévias ou de instalação - e um formulário que objetivava elencar o número da condicionante, o resumo de cada uma delas e os códigos das disciplinas do curso de Engenharia Ambiental que mais se adequavam a execução da mesma, caso os integrantes fossem os profissionais responsáveis pela implementação e cumprimento destas condicionantes.

O preenchimento das informações solicitadas foi elaborado juntamente com a consulta à grade curricular do curso para que os discentes levassem em consideração não somente as disciplinas obrigatórias, mas também contemplassem as disciplinas eletivas disponibilizadas pela Universidade. Em posse dos dois documentos então, iniciou-se o processo de debate a cerca da temática proposta.

Os debates se sucederam conforme o esperado, levantando-se algumas discordâncias entre membros dos grupos, tendo em vista questões como: maior afinidade por algumas disciplinas ofertadas e maior absorção do conteúdo ministrado, percepção da ausência de disciplinas pertinentes na grade do curso e a carga horária de eletivas realizadas por alguns membros dando a eles maior conhecimento do assunto em detrimento dos outros, por exemplo. Estabeleceu-se um tempo total de atividade de 100 minutos sendo que o debate entre os membros do grupo durou cerca de 80 minutos para que o preenchimento fosse minucioso garantindo a qualidade e a veracidade dos dados do levantamento e os 20 minutos restantes foram destinados a determinar as três disciplinas mais conexas para com o empreendimento potencialmente poluidor em questão. Foram um total de 213 condicionantes 
avaliadas e após a realização dos debates e o preenchimento dos formulários por parte de cada grupo iniciou-se o processo de compilamento e análise dos dados obtidos com o levantamento.

\section{RESULTADOS E DISCUSSÕES}

A Tabela 2 mostra as disciplinas do curso de Engenharia Ambiental que mais se correlacionaram com os empreendimentos poluidores/degradadores, sob a ótica dos estudantes. A coluna da direita lista a quantidade de vezes que a disciplina foi lembrada pelos estudantes ao lerem o teor das condicionantes. Isso significa, por exemplo, que a disciplina BEV 208 - Ecossistemas foi associada 12 vezes com o empreendimento de Lavra e beneficiamento de cobre. Este levantamento mostra de uma forma simples, quais as habilidades necessárias aos alunos para cumprir a exigência imposta pelo Órgão Ambiental licenciador.

Tabela 2 - Listagem dos empreendimentos analisados neste trabalho e as três disciplinas que mais se destacam sob a ótica do discente.

\begin{tabular}{|c|c|c|}
\hline Empreendimento & Disciplina & $\begin{array}{l}\text { Condicionantes } \\
\text { correlacionadas }\end{array}$ \\
\hline \multirow[t]{3}{*}{$\begin{array}{l}\text { Lavra e Beneficiamento de } \\
\text { Cobre }\end{array}$} & BEV 208 - Ecossistemas & 12 \\
\hline & BEV 214 - Ecologia Básica & 11 \\
\hline & $\begin{array}{l}\text { AMB } 176 \text { - Avaliação de Impacto e } \\
\text { Licenciamento Ambiental }\end{array}$ & 10 \\
\hline \multirow[t]{3}{*}{ Dragagem Portuária } & BEV $208-$ Ecossistemas & 6 \\
\hline & BEV 214 - Ecologia Básica & 6 \\
\hline & BEV 208 - Ecologia Aquática & 6 \\
\hline \multirow[t]{4}{*}{ UHE Belo Monte } & DIR 751 - Direito Ambiental & 6 \\
\hline & $\begin{array}{l}\text { AMB } 176 \text { - Avaliação de Impacto e } \\
\text { Licenciamento Ambiental }\end{array}$ & 6 \\
\hline & CIV 272 - Hidrologia Aplicada & 5 \\
\hline & $\begin{array}{c}\text { CIV } 424 \text { - Gerenciamento de Recursos } \\
\text { Hídricos }\end{array}$ & 5 \\
\hline \multirow[t]{3}{*}{ Implantação Porto Central } & DIR 751 - Direito Ambiental & 19 \\
\hline & BEV 208 - Ecologia Aquática & 17 \\
\hline & BEV 214 - Ecologia Básica & 14 \\
\hline \multirow[t]{3}{*}{ Metro de São Paulo } & $\begin{array}{c}\text { CIV } 275 \text { - Tratamento de Resíduos } \\
\text { Sólidos Urbanos }\end{array}$ & 6 \\
\hline & DIR 751 - Direito Ambiental & 5 \\
\hline & GEO 116 - Cartografia Aplicada & 4 \\
\hline \multirow[t]{3}{*}{ Ramal Ferroviário } & BEV 208 - Ecossistemas & 7 \\
\hline & DIR 751 - Direito Ambiental & 7 \\
\hline & $\begin{array}{c}\text { AMB } 176 \text { - Avaliação de Impacto e } \\
\text { Licenciamento Ambiental }\end{array}$ & 6 \\
\hline \multirow[t]{3}{*}{$\begin{array}{c}\text { Gasoduto marítimo e } \\
\text { terrestre }\end{array}$} & $\begin{array}{c}\text { AMB } 102 \text { - Ocupação e Planejamento } \\
\text { Territorial }\end{array}$ & 6 \\
\hline & BEV 214 - Ecologia Básica & 6 \\
\hline & $\begin{array}{c}\text { AMB } 176 \text { - Avaliação de Impacto e } \\
\text { Licenciamento Ambiental }\end{array}$ & 6 \\
\hline
\end{tabular}


"Os desafios para formar hoje o engenheiro do amanhã"

\begin{tabular}{|c|c|c|}
\hline Estrada de rodagem & BEV 214 - Ecologia Básica & 6 \\
& AMB 307 - Contaminação e Remediação & 6 \\
de Solos e Água Subterrânea. & MIN 215-Geotecnia & 5 \\
\hline
\end{tabular}

Fonte: Os autores.

Este levantamento mostrado na Tabela 2 possibilitou a elaboração de um ranking das dez matérias que mais apareceram nas respostas dadas pelos discentes quanto à viabilidade de execução das condicionantes impostas pelos empreendimentos estudados. Tal ranking é mostrado na Figura 1, em que se identificou o número de vezes em que a disciplina foi citada na pesquisa. Nota-se um destaque para as disciplinas da área de ciências biológicas, tanto pelo maior número de ocorrências quanto pelo maior número de disciplinas correlatas segundo os discentes.

Figura 1 - Ranking das 10 disciplinas do curso de Engenharia Ambiental da UFOP que mais foram identificadas pelos alunos como necessárias para o atendimento de condicionantes.

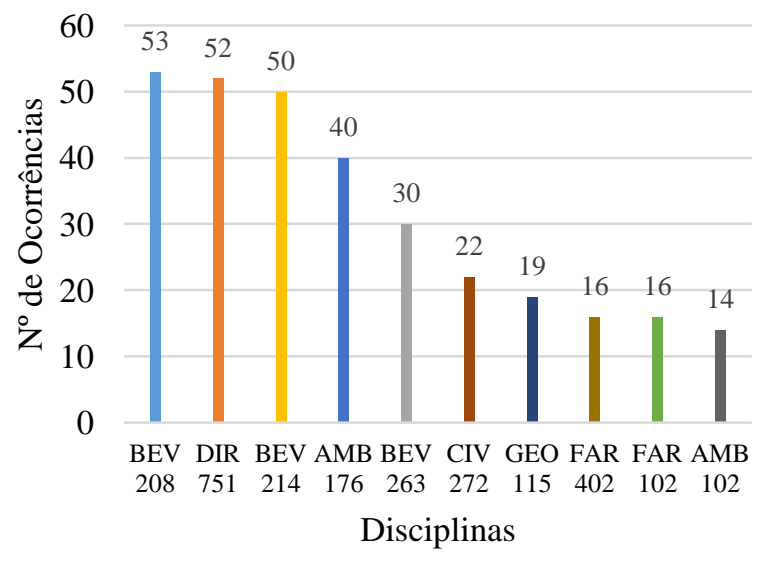

Fonte: Os autores.

\author{
- AMB 176 Avaliação de Impacto e Lic. Ambiental \\ DIR 751 Direito Ambiental \\ BEV 263 Ecologia Aquática \\ BEV 214 Ecologia Básica \\ - BEV 208 Ecossistemas \\ FAR 102 Epidemiologia Ambiental \\ - GEO 115 Erosão e Deposição de Sedimentos \\ - CIV 272 Hidrologia Aplicada \\ - AMB 102 Ocupação e Planejamento Territorial \\ - FAR 402 Qualidade de Águas
}

A Figura 1 é capaz ainda de denotar também a correlação entre disciplinas uma vez que para o processo de licenciamento, independentemente do empreendimento em questão, entende-se por parte do aluno que a identificação e caracterização adequada do meio bem como o conhecimento aprofundado das legislações vigentes são consideradas como "carrochefe" para a boa execução de qualquer projeto ambiental. Isso é evidenciado pelo maior número de ocorrências das disciplinas de Ecossistemas (BEV 208), Ecologia (BEV 214), Direito Ambiental (DIR 751), e Avaliação de Impacto e Licenciamento Ambiental (AMB 176). Fica clara a variabilidade de disciplinas necessárias para a atuação competente dos engenheiros ambientais no processo de implementação de obras dos segmentos analisados e a interdisciplinaridade do curso. Salienta-se que todas as outras disciplinas, mesmo com menor ocorrência, também são de suma importância e complementam o processo de licenciamento. Além disso, a listagem de disciplinas mais relevantes pode variar em função dos empreendimentos analisados.

Outro dado importante obtido através desta pesquisa foi a ocorrência de disciplinas comuns a todas as licenças avaliadas como mostra a Tabela 3. Este resultado corrobora com o citado acima a cerca de disciplinas-base para o licenciamento ambiental e indica um resultado significativo tendo em vista que a disciplina "FAR102 - Qualidade de Águas" não é obrigatória e segundo os discentes a mesma é indispensável não apenas para os processos de 
licenciamento, mas para a atuação profissional em diversas vertentes do mercado de trabalho. A Tabela 3 mostra ainda que disciplinas de diversos Departamentos da Universidade são bastante relevantes para o curso, desde a Biologia (código BEV), Geologia (código GEO), Farmácia (código FAR) e o Departamento de Engenharia Ambiental (código AMB). Isso indica o teor transdisciplinar do curso.

Tabela 3 - Listagem das disciplinas correlacionadas a todas as licenças.

\begin{tabular}{|l|l|l|}
\hline \multicolumn{1}{|c|}{ Código } & \multicolumn{1}{|c|}{ Disciplina } & $\begin{array}{l}\text { Condicionantes } \\
\text { correlacionadas }\end{array}$ \\
\hline BEV 208 & Ecossistemas & 53 \\
\hline BEV 214 & Ecologia Básica & 50 \\
\hline AMB 176 & Avaliação de Impacto e Licenciamento Ambiental & 40 \\
\hline GEO 115 & Erosão e Deposição de Sedimentos & 19 \\
\hline FAR 402 & Qualidade de Águas & 16 \\
\hline
\end{tabular}

Fonte: Os autores.

Quanto ao questionamento das necessidades de outras disciplinas para o cumprimento das condicionantes ambientais, foram apontadas lacunas em diversos aspectos, sendo as principais, a oferta de disciplinas de forma anual, o que pode inviabilizar o discente de cursála por conflitos de horário e carga horária do período; a ausência de vagas nas disciplinas eletivas pertinentes; o enfoque específico em determinadas temáticas que deixam de abranger outros setores do mercado e até mesmo a ausência na grade curricular como mostra a Tabela 4. Note que as disciplinas sem código não fazer parte da grade curricular do curso, mas podem ajudar a guiar possíveis melhorias no plano pedagógico do curso.

Tabela 4 - Listagem de necessidades apontadas pelos alunos para atendimento às condicionantes das licenças ambientais

\begin{tabular}{|l|l|}
\hline \multicolumn{1}{|c|}{ Código } & \multicolumn{1}{c|}{ Disciplina } \\
\hline- & Monitoramento Ambiental \\
\hline- & Emissão e Neutralização de Ruídos \\
\hline- & Recuperação de Áreas Degradadas (enfoque geral) \\
\hline BEV 263 & Ecologia Aquática \\
\hline- & Tratamento de Resíduos \\
\hline AMB 307 & Contaminação e Remediação: Solo e Água Subt. \\
\hline CIV 424 & Gerenciamento de Recursos Hídricos A \\
\hline
\end{tabular}

Fonte: Os autores.

\section{CONSIDERAÇÕES FINAIS}

Neste trabalho, a interdisciplinaridade do curso de Engenharia Ambiental da UFOP foi avaliada sob a ótica do estudante de graduação. Alunos da disciplina AMB 176 - Avaliação de Impacto e Licenciamento Ambiental fizeram uma dinâmica de grupo para correlacionar as disciplinas do curso com as condicionantes ambientais de 8 empreendimentos potencialmente poluidores/degradadores. As principais conclusões do trabalho são resumidas a seguir:

i. Disciplinas da área de ciências biológicas BEV 208 Ecossistemas e BEV 214 - Ecologia, e da área de gestão ambiental DIR 751 - Direito Ambiental e AMB 176 - Avaliação de Impacto e Licenciamento Ambiental foram apontadas como mais relevantes pelos discentes. 
ii. Dentre as sete necessidades apontadas pelos alunos como pré-requisitos para o atendimento de condicionantes ambientais, quatro delas ainda não são ofertadas pelo curso, mas podem ser futuramente incluídas no plano pedagógico.

iii. As cinco disciplinas do curso relevantes (comuns) para todos os empreendimentos são oferecidas por diversos departamentos dentro da Universidade, refletindo o caráter transdisciplinar do curso de Engenharia Ambiental.

iv. Das cinco disciplinas do curso correlacionadas a todas as licenças ambientais, a disciplina FAR 402 - Qualidade das Águas atualmente é eletiva no curso de Engenharia Ambiental.

\section{Agradecimentos}

Os autores agradecem o suporte da Universidade Federal de Ouro Preto para a elaboração do presente trabalho.

\section{REFERÊNCIAS}

DE ARAÚJO, Rafael Gonçalves Bezerra et al. Developing professional and entrepreneurship skills of engineering students through problem-based learning: a case study in brazil. The International journal of engineering education, v. 36, n. 1, p. 155-169, 2020.

BURNLEY, Stephen; WAGLAND, Stuart; LONGHURST, Phil. Using life cycle assessment in environmental engineering education. Higher Education Pedagogies, v. 4, n. 1, p. 64-79, 2019.

BUTKUS, Michael A.; KELLEY, Michael B. Approach for integrating professional practice issues into undergraduate environmental engineering design projects. Journal of Professional Issues in Engineering Education and Practice, v. 130, n. 3, p. 166-172, 2004.

FOLEY, Greg. Reflections on interdisciplinarity and teaching chemical engineering on an interdisciplinary degree programme in biotechnology. Education for Chemical Engineers, v. 14, p. 35-42, 2016.

FORTUIN, K. P. J.; VAN KOPPEN, C. S. A.; LEEMANS, R. The Value of Conceptual Models in Coping with Complexity and Interdisciplinarity in Environmental Sciences Education. Bioscience, v.61, n.10, p. 802-814, 2011.

GUIDER, F. et al. Implementation of a Multidisciplinary Professional Skills Course at an Electrical Engineering School. IEEE Transactions on Education, v.55, n.3, p. 332-340, 2012.

HARRISON, G. P.; MACPHERSON, D.E.; WILLIAMS, D.A. Promoting interdisciplinarity in engineering teaching. European Journal of Engineering Education, v.32, n.3, p. 285293, 2007.

LU, M. Integrating Sustainability into the Introduction of Environmental Engineering. Journal of Professional Issues in Engineering Education and Practice, v.141, n.2, C5014004, 2015. 
RICKLES, P.; ELLUL, C.; HAKLAY, M. A suggested framework and guidelines for learning GIS in interdisciplinary research. Geo: Geography and Environment. v.4, n.2, e00046, 2017.

RODRIGUES, G. S. S. C. A análise interdisciplinar de processos de Licenciamento Ambiental no estado de Minas Gerais: conflitos entre velhos e novos paradigmas. Sociedade \& Natureza, v.22, n.2, p. 267-282, 2010.

SEMERJIAN, J. et al. Interdisciplinary Approach to Environmental Education. Journal of Professional Issues in Engineering Education and Practice, v.130, n.3, p. 173-181, 2004.

ZHANG, J. et al. Exploring an Interdisciplinary BIM-Based Joint Capstone Course in Highway Engineering. Journal of Civil Engineering Education, v.146, n.3, p. 05020004, 2020 .

\title{
THE INTERDISCIPLINARITY OF ENVIRONMENTAL ENGINEERING FROM THE PERSPECTIVE OF UNDERGRADUATE STUDENT: CORRELATING COURSE SUBJECTS WITH ENVIRONMENTAL CONDITIONS.
}

\begin{abstract}
Engineering courses are essentially interdisciplinary, reflecting the needs and challenges of conducting complex real-life projects. Environmental Engineering is one of those interdisciplinarity reliant courses among engineering graduation programs. In this paper, students from the Impact Assessment and Environmental Licensing class (AMB 176), from the Environmental Engineering course at the Federal University of Ouro Preto (UFOP), survey how the classes offered by the program relate to the environmental conditions imposed by the licensing processes. Environmental conditions of eight different projects subjected to an Environmental Impact Study (EIA) according to CONAMA Resolution 01/86 were evaluated. Survey participants were instructed to build a connection between the mandatory and elective classes required to meet the demands of licensing agencies. Results have shown that classes in the areas of biological sciences and environmental management were pointed out as more relevant by the students. Four out of the seven needs identified by students as prerequisites for meeting environmental conditions are not yet offered by the course. Five relevant (common) course classes for all projects are offered by different departments within the University, reflecting the previously mentioned interdisciplinary nature of the Environmental Engineering course. Analyzing the five course subjects related to all environmental licenses, the Water Quality class (FAR 402) is currently non-mandatory (elective) in the Environmental Engineering course.
\end{abstract}

Keywords: Interdisciplinarity. Environmental Engineering. Environmental Licensing. Environmental Conditions. 